\title{
Discretion Rather Than Rules: Ramsey Policy as the Unique Outcome
}

\section{Chihiro Morooka}

Faculty of Economics, The University of Tokyo, Tokyo, Japan

Email: morooka.chihiro@mail.u-tokyo.ac.jp

How to cite this paper: Morooka, C (2020). Discretion Rather Than Rules: Ramsey Policy as the Unique Outcome. Theoretical Economics Letters, 10, 1199-1204. https://doi.org/10.4236/tel.2020.106071

Received: October 19, 2020

Accepted: November 27, 2020

Published: November 30, 2020

Copyright (c) 2020 by author(s) and Scientific Research Publishing Inc. This work is licensed under the Creative Commons Attribution International License (CC BY 4.0).

http://creativecommons.org/licenses/by/4.0/

\begin{abstract}
This paper analyzes finitely repeated policy games where the government and the private sector alternately revise their actions from the set of non-negative real numbers. Unlike previous studies on policy games, the one-shot inefficient Nash equilibrium, known as the Kydland-Prescott outcome, is avoided and only the optimal Ramsey outcome is established in subgame perfect equilibria. Therefore, the Ramsey policy is time-consistent in our model, whereas the Kydland-Prescott outcome is not.
\end{abstract}

\section{Keywords}

Macroeconomic Dynamics, Inflation Targeting, Time Consistency, Ramsey Policy, Alternately Repeated Games, Anti-Folk Theorem

\section{Introduction}

In macroeconomic theory, the fundamental problem of implementing the optimal policy arises when the government does not have credible technology of commitment, and the private sector has rational expectations about the government's policy. To analyze this problem, policy games between the government and the private sector are considered. In policy games, it is known that only one inefficient Nash equilibrium exists, known as the Kydland-Prescott (KP) outcome, because the government has a dominant myopic action, leading to the private sector's inefficient response. This outcome is Pareto-dominated by the Ramsey outcome, which maximizes the government's objective function by behaving as the Stackelberg leader.

Avoiding the KP outcome requires a long-run trustful relationship, which is often modeled by repeated games. Previous research in this field includes Chari and Kehoe (1990) and Stokey (1991). However, these studies only demonstrated the existence of efficient subgame perfect equilibria. In their models, the KP 
outcome is also subgame perfect. Indeed, the folk theorem in infinitely repeated games guarantees that payoffs between the Ramsey and the KP outcomes can be obtained in equilibria; as Sargent (1999) points out, the pessimism of Kydland and Prescott (1977) was replaced with multiple equilibria.

Many studies on repeated policy games, including the abovementioned, assume the participants' simultaneous decision-making. However, when we investigate the long-run relationship between the participants, it seems more natural to assume that they decide actions asynchronously (e.g., Lagunoff \& Matsui, 1997). For instance, the private sector needs a significant amount of time to upgrade their production equipment, and the government requires years to revise the laws once decided upon, making their decisions alternate.

We examine whether players change their behavior in repeated policy games when the repetition becomes finite and alternate, with the assumption that, similar to the models of policy games in many other studies, the players' action sets are in continuum (i.e., each player chooses one non-negative real number). ${ }^{1} \mathrm{We}$ demonstrate that only the efficient result arises; when the number of repetitions is not too small, the government plays the Pareto-optimal action in every period, and the private sector responds to it in the best way. Thus, our results establish a version of anti-folk theorems.

The proof requires backward induction. Suppose that the government moves in the terminal round. ${ }^{2}$ It is certain that the government plays a myopic action at this stage. Then, consider the private sector's final move, dating back one period. When the government's current action is myopic, the private sector chooses the best response, and the government's total payoff is low. On the other hand, if the government's current action is efficient, the private sector's response becomes similar to the mean value of the Ramsey and KP outcomes, raising the government's total payoff for these two periods. Consequently, it is optimal for the government to choose an efficient action throughout the play, restoring the time consistency of the Ramsey outcome.

In the models of macroeconomic dynamics, it has been observed by previous studies, especially Libich and Nguyen (2013), that the timing of participants' opportunity to change actions, which determines the degree of their commitment power, has a significant influence on which of them is better off. Our research focuses on, in which dynamic decision-making structure, both participants can obtain relatively high payoffs that are Pareto-optimal. In research on repeated policy games, Cho and Matsui (1995) were the first to demonstrate that only the efficient result appears in equilibria. However, they assumed that the private sector is distributed over a unit interval and that it has limited rationality. Following this result, Cho and Matsui (2005) presented efficient results under full rationality of the private sector in alternately repeated games, which have

${ }^{1}$ In the case where players have only a finite number of actions, see previous work by the author, Morooka (2020). There, it is shown that an efficient equilibrium exists in a specific $2 \times 3$ game with finite alternating repetition, which can be viewed as a repeated policy game.

${ }^{2}$ Our result holds regardless of the player who moves in the first and last periods. 
been typical models of asynchronous decision-making used in the extensive literature on repeated games since Maskin and Tirole (1988). However, in Cho and Matsui (2005), the continuum of the private sector was still assumed; with a finite number of private sectors, the stage game satisfies the full dimensionality condition and the folk theorem in asynchronously repeated games by Yoon (2001) holds.

There is also a connection between our study and previous research on finitely repeated games with multiple one-shot Nash equilibria. In simultaneous move games, Benoit and Krishna (1982) proved the folk theorem with multiple one-shot equilibria yielding different payoffs. In finitely repeated common-interest games with asynchronous moves which possibly involve multiple Pareto-ranked Nash equilibria, Dutta (2012) proved that the Pareto-dominating Nash equilibrium is played in almost all periods in subgame perfect equilibria. Unlike these results, we focus on the stage game with only one Nash equilibrium yielding inefficient payoffs and show that the efficient actions are played in almost every period of its finite repetition.

The remainder of this paper is organized as follows. In Section 2, we define the model and present the results. We present a discussion and concluding remarks in Section 3.

\section{Policy Games}

Let $I=\{$ the government, the private sector $\}$ be the set of players. We define the one-shot payoff functions of the government $\left(u_{g}\right)$ and private sector $\left(u_{p}\right)$, as follows:

$$
\begin{gathered}
u_{g}\left(\pi, \pi^{e}\right)=\left(\min \left\{\pi^{e}, \bar{\pi}\right\}+\alpha\right) \min \{\pi, 2 \bar{\pi}-\pi\}-\beta \pi^{e}, \\
u_{p}\left(\pi, \pi^{e}\right)=-\left(\pi^{e}-\pi\right)^{2} .
\end{gathered}
$$

Here, each action of $\pi$ and $\pi^{e}$ in $[0,+\infty)$ is the government's inflation target and private sector's expectation, respectively. The parameters, $\alpha>0$, $\beta>0$, and $\bar{\pi}>0$ are given exogenously with $\bar{\pi}+\alpha-\beta<0$.

We briefly explain these functions. The private sector wants to set $\pi^{e}$ to the same rate as the government's inflation rate $\pi$. The government receives a damage in proportion to the private sector's rate of expectation but wants to set the inflation rate to a myopic value $\bar{\pi}$. The government's decision also has a positive correlation with the private sector's expectation when the expectation is not too high. There is only one Nash equilibrium $\left(\pi, \pi^{e}\right)=(\bar{\pi}, \bar{\pi})$ with the government's payoff $(\bar{\pi}+\alpha-\beta) \bar{\pi}<0$, while if $\left(\pi, \pi^{e}\right)=(0,0)$ is played, the government's payoff is zero and Pareto-optimal.

For $i \in I$ and $T \in \mathbb{N}$, the alternately repeated game $G(i, T)$ with perfect monitoring is played in periods $t=0,1,2, \cdots, T$. Player $i$ can revise the action only in even periods, including period zero, while $i$ s opponent can revise the action only in odd periods. Players receive one-shot payoffs without discounting at 
the end of every period $t \geq 1$, depending on the actions played in that period. In period zero, it is assumed that only $i$ can move and that players receive no payoff.

We prove the following theorem, which states that the Pareto-optimal actions are played throughout the repetition if the government's damage from the private sector's high rate of expectation is relatively large.

Theorem 1. If $\beta>2 \alpha+2.5 \bar{\pi}$ holds, then in every period $t \leq T-2$, $\left(\pi, \pi^{e}\right)=(0,0)$ is played on the path in any subgame perfect equilibrium of $G(i, T)$ with $i \in I$ and $T \geq 3$.

Proof. We use backward induction and consider only the case in which the government moves in period $T^{3}$ For $s \in\{1, \cdots, T+1\}$, the period $T-s+1$ is referred to as "the $s$-th stage."

Step 1. In the first stage, the government always plays $\pi=\bar{\pi}$.

Proof of Step 1. This step holds because $\bar{\pi}$ is the government's strictly dominant action.

Step 2. In the second stage, the private sector plays $\pi^{e}=0.5\left(\pi_{3}+\bar{\pi}\right)$ when the government's current action is $\pi_{3}$. Therefore, the equilibrium path from this stage is $\left(\pi_{3}, 0.5\left(\pi_{3}+\bar{\pi}\right)\right) \rightarrow\left(\bar{\pi}, 0.5\left(\pi_{3}+\bar{\pi}\right)\right)$.

Proof of Step 2. If the private sector plays $\pi^{e}$, its continuation payoff is $-\left(\pi^{e}-\pi_{3}\right)^{2}-\left(\pi^{e}-\bar{\pi}\right)^{2}$, which is maximized at $\pi^{e}=0.5\left(\pi_{3}+\bar{\pi}\right)$.

Step 3. In the third stage, the government's action $\pi>\bar{\pi}$ is strictly dominated by $\pi=\bar{\pi}$ for any current action of the private sector.

Proof of Step 3. For any $k>0$ and the private sector's current action $\pi_{4}^{e}$, the government's continuation payoff when it plays $\bar{\pi}+k$ is at most as follows:

$$
\begin{aligned}
& \min \left\{\pi_{4}^{e}, \bar{\pi}\right\}(\bar{\pi}-k)+\alpha(\bar{\pi}-k)-\beta \pi_{4}^{e}+\bar{\pi}(\bar{\pi}-k)+\alpha(\bar{\pi}-k) \\
& -\beta(\bar{\pi}+0.5 k)+\bar{\pi}^{2}+\alpha \bar{\pi}-\beta(\bar{\pi}+0.5 k) .
\end{aligned}
$$

On the other hand, when the government plays $\bar{\pi}$, its continuation payoff is as follows:

$$
\min \left\{\pi_{4}^{e}, \bar{\pi}\right\} \bar{\pi}+3 \alpha \bar{\pi}-\beta \pi_{4}^{e}+2 \bar{\pi}^{2}-2 \beta \bar{\pi} .
$$

We can calculate that $(4)-(3)=\left(\beta+2 \alpha+\bar{\pi}+\min \left\{\pi_{4}^{e}, \bar{\pi}\right\}\right) k>0$.

Step 4 . In the third stage, the government always plays $\pi=0$. Therefore, for the private sector's current action $\pi_{4}^{e}$, the path is $\left(0, \pi_{4}^{e}\right) \rightarrow(0,0.5 \bar{\pi}) \rightarrow(\bar{\pi}, 0.5 \bar{\pi})$.

Proof of Step 4. If the government plays $\pi \leq \bar{\pi}$, its continuation payoff is as follows:

$$
\begin{aligned}
& \left(\min \left\{\pi_{4}^{e}, \bar{\pi}\right\}+\alpha\right) \pi-\beta \pi_{4}^{e}+(0.5 \pi+0.5 \bar{\pi}+\alpha) \pi-\beta(0.5 \pi+0.5 \bar{\pi}) \\
& +(0.5 \pi+0.5 \bar{\pi}+\alpha) \bar{\pi}-\beta(0.5 \pi+0.5 \bar{\pi}) .
\end{aligned}
$$

This is a quadratic function of $\pi$, and the value is $-\beta \pi_{4}^{e}+\bar{\pi}(-\beta+0.5 \bar{\pi}+\alpha)$ ${ }^{3}$ That is, in either case ( $i=$ the government, $T$ is even) or ( $i=$ the private sector, $T$ is odd). The proof is analogous in the other cases. 
at $\pi=0$, whereas it is $-\beta \pi_{4}^{e}+\bar{\pi}\left(-2 \beta+2 \bar{\pi}+\min \left\{\pi_{4}^{e}, \bar{\pi}\right\}+3 \alpha\right)$ at $\pi=\bar{\pi}$. When $\beta>2 \alpha+2.5 \bar{\pi}$ holds, the value at $\pi=0$ is greater than the value at $\pi=\bar{\pi}$ independent of the private sector's current action, which means that it is strictly optimal for the government to play $\pi=0$ in this stage.

Step 5. In the fourth stage, the private sector plays $\pi^{e}=0.5 \pi_{5}$ when the government's current action is $\pi_{5}$. Therefore, the path is $\left(\pi_{5}, 0.5 \pi_{5}\right) \rightarrow\left(0,0.5 \pi_{5}\right) \rightarrow(0,0.5 \bar{\pi}) \rightarrow(\bar{\pi}, 0.5 \bar{\pi})$.

Step 6 . In the fifth stage, the government always plays $\pi=0$. Therefore, for the private sector's current action $\pi_{6}^{e}$, the path is $\left(0, \pi_{6}^{e}\right) \rightarrow(0,0) \rightarrow(0,0) \rightarrow(0,0.5 \bar{\pi}) \rightarrow(\bar{\pi}, 0.5 \bar{\pi})$

We omit the proof of these steps because it uses the same logic as that of Steps 2,3 , and 4 .

Step 7. In the $s$-th stage with $s \geq 6$, the private sector plays $\pi^{e}=0.5 \pi_{s+1}$ when the government's current action is $\pi_{s+1}$, and the government always plays $\pi=0$.

The proof of this step is given by a mathematical induction from the fourth stage, dating backward by one period, which ends at period zero. In the fourth and fifth stages, the corresponding actions above are optimal, as shown in Steps 5 and 6 . For the $2 s$-th stage with $s \geq 3$ (the private sector's node), we can demonstrate that its action is optimal by the logic analogous to Step 2. For the $2 s+1$-th stage with $s \geq 3$ (the government's node), the optimality is also shown by the analogous logic with Steps 3 and 4. Finally, in period zero, player $i$ plays zero, completing the proof of Theorem 1 .

\section{Discussion and Conclusion}

We analyzed policy games with finite alternating repetitions. In the model of dynamic policy games, no payoff-relevant links between periods result in the folk-like theorem. On the other hand, when repetition becomes alternate, policy games sometimes have a sharp prediction. We can eliminate the inefficient KP outcome from the set of equilibria. Thanks to a payoff-relevant link between periods, the government can commit to a lower inflation rate and influence the private sector's response. In future research, we will generalize the structure of asynchronous decision-making processes to investigate the robustness of our results.

\section{Acknowledgements}

I would like to thank Ryo Jinnai, Akihiko Matsui, and Yasuyuki Miyahara, and those attending my presentation at the Japanese Economic Association 2020 Autumn Meeting at Rissho University for their useful comments and discussions. I also acknowledge helpful comments and suggestions by referees. This research did not receive any specific grant from funding agencies in the public, commercial, or not-for-profit sectors. 


\section{Conflicts of Interest}

The author declares no conflicts of interest regarding the publication of this paper.

\section{References}

Benoit, J., \& Krishna, V. (1982). Finitely Repeated Games. Econometrica, 53, 905-922. https://doi.org/10.2307/1912660

Chari, V., \& Kehoe, P. J. (1990). Sustainable Plans. Journal of Political Economy, 98, 783-802. https://doi.org/10.1086/261706

Cho, I., \& Matsui, A. (1995). Induction and the Ramsey Policy. Journal of Economic Dynamics and Control, 19, 1113-1140. https://doi.org/10.1016/0165-1889(94)00821-X

Cho, I., \& Matsui, A. (2005). Time Consistency in Alternating-Move Policy Games. Japanese Economic Review, 56, 273-294. https://doi.org/10.1111/j.1468-5876.2005.00326.x

Dutta, P. (2012). Coordination Need Not Be a Problem. Games and Economic Behavior, 76, 519-534. https://doi.org/10.1016/j.geb.2012.07.002

Kydland, F., \& Prescott, E. C. (1977). Rules Rather than Discretion: The Inconsistency of Optimal Plans. Journal of Political Economy, 85, 473-491.

https://doi.org/10.1086/260580

Lagunoff, R., \& Matsui, A. (1997). Asynchronous Choice in Repeated Coordination Games. Econometrica, 65, 1467-1477. https://doi.org/10.2139/ssrn.66488

Libich, J., \& Nguyen, D. T. (2013). Macro Meets Micro: Stochastic (Calvo) Revisions in Games. The B.E. Journal of Theoretical Economics, 14, 339-369. https://doi.org/10.1515/bejte-2013-0042

Maskin, E., \& Tirole, J. (1988). A Theory of Dynamic Oligopoly II: Price Competition, Kinked Demand Curves, and Edgeworth Cycles. Econometrica, 56, 571-599. https://doi.org/10.2307/1911701

Morooka, C. (2020). Efficiency in a Policy Game with Finite Alternating Repetition. The Economic Review, 71, 275-278. https://hdl.handle.net/10086/31309

Sargent, T. J. (1999). The Conquest of American Inflation. Princeton, NJ: Princeton University Press. https://doi.org/10.1515/9780691186689

Stokey, N. L. (1991). Credible Public Policy. Journal of Economic Dynamics and Control, 15, 627-656. https://doi.org/10.1016/0165-1889(91)90036-Z

Yoon, K. (2001). A Folk Theorem for Asynchronously Repeated Games. Econometrica, 69, 191-200. https://doi.org/10.1111/1468-0262.00182 